\title{
Transition from child and adolescent to adult mental health services in the Republic of Ireland: An investigation of process and operational practice
}

\author{
Niamh McNamara ${ }^{1}$, Fiona McNicholas* 1,2, Tamsin Ford ${ }^{3}$, Moli Paul ${ }^{4}$, Blanaid Gavin ${ }^{2}$, Imelda \\ Coyne $^{5}$, Walter Cullen ${ }^{6}$, Karen O'Connor ${ }^{7}$, Nicolas Ramperti ${ }^{8}$, Barbara Dooley ${ }^{9}$, Siobhan \\ Barry $^{10}$, Swaran P Singh ${ }^{4}$
}

${ }^{1}$ School of Medicine \& Medical Science, University College Dublin, Ireland

${ }^{2}$ Lucena Clinic, Rathgar, Dublin, Ireland

${ }^{3}$ Peninsula College of Medicine \& Dentistry, Universities of Exeter \& Plymouth, UK

${ }^{4}$ Warwick Medical School, University of Warwick, UK

${ }^{5}$ School of Nursing \& Midwifery, Trinity College Dublin, Ireland

${ }^{6}$ Graduate School of Medicine, University of Limerick, Ireland

${ }^{7}$ St John of God Hospital, Dublin, Ireland

${ }^{8}$ Ballyfermot Mental Health Centre, Dublin, Ireland

${ }^{9}$ School of Psychology, University College Dublin, Ireland

${ }^{10}$ Cluain Mhuire Mental Health Service, Dublin, Ireland

Running Title: Investigating operational practice in transition

*Corresponding Author: Professor Fiona McNicholas, Consultant Child \& Adolescent

Psychiatrist, Lucena Clinic, 59 Orwell Road, Rathgar, Dublin 6, Ireland

Email: fiona.mcnicholas@sjog.ie

Tel.: +35314923596

Fax: +35314923823 


\begin{abstract}
Aim: Ensuring a seamless transition from child to adult mental health services poses challenges for services worldwide. This is an important process in the on-going care of young people with mental illness; therefore it is incumbent on all countries to probe their individual structures to assess the quality of mental health service delivery to this vulnerable cohort. To date, there have been no published studies on the transition from Child to Adult Mental Health Services in the Republic of Ireland. To this end, a nationwide survey of transition policies of community mental health teams in both services was conducted in order to compare best practice guidelines for transition with current process and experience in clinical practice. Method: Structured interviews were conducted with 57 consultant psychiatrists (representing 32 CAMHS teams and 25 AMHS teams) to obtain information on annual transition numbers, existing transition policies, and operational practice from the professional perspective. Results: Numbers of young people considered suitable for transfer to adult services $(M=7.73, S D=9.86, n=25)$ were slightly higher than numbers who actually transferred $(M=4.50, S D=3.33, n=20)$. There is a lack of standardised practice nationwide regarding the service transition boundary, an absence of written transition policies and protocols, and minimal formal interaction between child and adult services. Conclusions: The findings suggest that there are critical gaps between current operational practice and best practice guidelines. Future studies will investigate the impact this has on the transition experiences of young people, their carers and healthcare professionals.
\end{abstract}

Keywords: adolescence, good practice, mental health services, service development, transition to adult care 


\section{Transition from child and adolescent to adult mental health services in the Republic of Ireland: An investigation of process and operational practice}

\section{INTRODUCTION}

Globally, the mental health of adolescents, particularly those entering adulthood, is declining. ${ }^{1}$ Adolescents' psychological needs are greatest during the developmental transition to adulthood but paradoxically, the point of service transition from Child \& Adolescent Mental Health Services (CAMHS) to Adult Mental Health Services (AMHS) can be where services are at their weakest. ${ }^{2}$ In mental healthcare, transition is a distinct concept from transfer. ${ }^{3}$ Transfer is likened to an event involving the termination of the young person's care in CAMHS and its re-establishment in AMHS. ${ }^{3}$ In comparison, transition is a planned process that addresses a range of therapeutic and developmental needs. ${ }^{4}$

Transition is a challenging issue for mental health services worldwide. ${ }^{5,6}$ Service configurations in many countries may create certain barriers to successful transition, including: arbitrarily drawn service boundaries, ${ }^{7,8}$ differing service thresholds, ${ }^{9,10}$ and the different contexts (and cultures) in which child and adult services operate ${ }^{11,12}$. Consequently, the transition process is frequently experienced negatively by young people, carers, and professionals. ${ }^{11,13}$

Importantly, negative transition experiences can adversely impact young people's future service engagement ${ }^{6}$ and can reverse any health gains made in CAMHS. ${ }^{8,14}$ A large-scale US study revealed a $50 \%$ decline in service usage at the age of transition. ${ }^{15}$ That this decrease in service use occurs at this time suggests that poor engagement may be linked with problems experienced during transition. ${ }^{7}$ Young people who dis-engage from services frequently reengage later during crises, which might be prevented by ensuring continuous care and support. ${ }^{5}$ 
The UK 'TRACK' study, ${ }^{9}$ is one of the few studies to examine the transition experiences of young people, carers, and professionals. Through a case note review and detailed interviews, Singh and colleagues ${ }^{9}$ found that of a sample of 90 young people, only 4 experienced an optimal transition defined as fulfilling four criteria: continuity of care (engaged in AMHS three months post transition or appropriately discharged), at least one transition planning meeting, good information transfer, and a period of parallel care. Successful transitions were described as gradual processes that were tailored to individual needs, while suboptimal transitions were hindered by poor inter-agency communication and a lack of flexibility in service provision. ${ }^{9,16}$ Similar transition problems have been reported in the US, Canada, and Australia. ${ }^{9,15,17}$

Youth mental health is a global priority and it is incumbent on all countries to probe their individual structures and service provision for those in transition. In this paper, we report the findings of Stage 1 of the ITRACK study, the first systematic examination of transition in the Republic of Ireland. Understanding this process is a necessary step towards improving the quality of the mental health service delivered to young people and ultimately promoting recovery.

\section{Mental Health Services for Young People in the Republic of Ireland}

Mental health services are primarily provided for young people in the Republic of Ireland by publicly funded CAMHS teams (At the time this study was conducted there were 56 teams in place). Most accept new referrals up to age 16 and those requiring on-going care are transferred to AMHS at 18. Like CAMHS, the community mental health team, led by a consultant psychiatrist, is the standard service configuration in AMHS and there are approximately 126 of these nationwide. Teams can develop their own protocols for clinical and operational practice to meet the needs of the population they serve. ${ }^{18}$ CAMHS and AMHS teams are not co-terminus. 
The Irish Working Group on Child and Adolescent Psychiatric Services ${ }^{19}$ has provided recommendations on ensuring seamless transitions between services including: prioritising continuity of care, ensuring flexibility in line with individual needs, and developing structures with input from both services with particular emphasis on planning and joint working. Although it is believed that transition is not always positively experienced by Irish young people $^{19}$, there is limited evidence about the nature and magnitude of the problem and how it might be addressed. This paper reports the findings of a nationwide survey of CAMHS and AMHS transition policies and procedures, the aim of which was to: (1) understand the process of mental health transition in the Republic of Ireland from the professional perspective and (2) compare best practice guidelines for transition with current process and experience in clinical practice. 


\section{METHOD}

\section{Sample}

Ethical approval was granted by relevant local ethics committees. In the absence of national protocols, individual teams have great flexibility in determining protocols for clinical and operational practice in transition. These protocols were the focus of study. A comprehensive national list of lead clinicians for CAMHS (61) and AMHS (146) teams was generated using information obtained from the Irish Health Services Executive website and telephoning individual clinics. Consultants from both public and private services were invited to participate.

\section{The ITRACK Questionnaire}

A two-part service mapping tool was developed based on the TRACK questionnaire. ${ }^{13}$ Part 1 referred to staffing levels, catchment population, and written transition policies. Part 2 referred to criteria determining service boundaries and annual transfer numbers. CAMHS clinicians were asked for the annual numbers considered suitable for transfer to AMHS and for annual numbers remaining in CAMHS past the transitional boundary along with the criteria used to determine both. To ascertain whether clinicians followed best practice, a list of questions was created based on best practice guidelines for $\operatorname{transition}^{20}$ (For the full list of items see Tables 1 and 2). Clinicians were asked to indicate whether each practice always, sometimes, or never happens.

\section{Data Collection and Analysis}

Clinicians were invited to participate by post. Reminder email requests, supplemented by telephone calls, were sent to improve recruitment rates. Informed consent was obtained from all participants. Structured telephone interviews were conducted using the study questionnaire. Descriptive statistics were derived on transition boundaries and practices for each team using SPSS. 


\section{RESULTS}

Data from 32 CAMHS, 31 general and one specialist team (52\% RR) and 25 AMHS (general) teams (17\% RR) were collected for this study. CAMHS catchment populations ranged from 60,000 to $1,200,000$ and whole-time equivalent staff ranged from 4 to $34.30(M=9.95$, $S D=6.95, N=23$ ). AMHS catchment populations ranged from 20,000 to 175,000 and WTE staff ranged from 4.25 to $37.90(M=12.60, S D=10.27, N=24)$. (Note that some AMHS clinicians provided staff numbers for their sector rather than their individual team).

\section{Age Boundaries}

27 CAMHS teams (84\%) reported 18 as the modal age boundary between CAMHS and AMHS; $4(13 \%)$ reported their upper age limit as 16, and $1(3 \%)$ reported an age limit of 17. These upper age limits applied primarily to young people who entered the service before the age of 16 . Only 10 teams (33\%) reported that they accepted new cases aged 16 to 18 (Four CAMHS teams did not respond to this question).

13 AMHS teams (52\%) reported a lower age limit of 18; 2 (8\%) reported a lower age limit of 17 and $10(40 \%)$ reported a lower age limit of 16. 21 AMHS teams (84\%) accept referrals of young people aged between 16 and 18 in instances where CAMHS did not accept new referrals in this age group, while 4 teams (16\%) reported that this never happened.

\section{Transfer Numbers}

Transfer numbers are based on clinician recall. CAMHS respondents estimated that the average annual number of cases that transferred to AMHS ranged from 1 to $10(M=4.50$, $S D=3.33, N=20$ ). AMHS estimated the average annual number of cases that transferred from CAMHS ranged from 0 to $10(M=4.82, S D=3.54, N=17)$. 


\section{Determining suitability for transfer to AMHS}

The average annual number of cases considered suitable for transfer to AMHS ranged between 1 and $50(M=7.73, S D=9.86, N=25)$. The factors CAMHS clinicians $(N=24)$ reported considering when deciding on suitability for transfer included: (1) diagnosis $(n=13)$; (2) the young person's/carers' preference $(n=10)$; (3) likely future need for psychiatric services $(n=9)$; and (4) an on-going need for medication $(n=4)$.

The average annual numbers of cases who remained in CAMHS beyond the transition boundary ranged from 0 to $21(M=5.46, S D=6.37, N=26)$. The main reasons for this were ( $N=27)$ : (1) supporting young people as they complete second level education $(n=15)$; (2) a diagnosis perceived not to meet AMHS diagnostic threshold (n=14); (3) completing a piece of therapeutic work $(n=10)$; (4) supporting the young person through other transitions (such as beginning university) ( $n=7)$; (5) the presence of a strong therapeutic relationship with a team member ( $n=5)$; and (6) awaiting an appointment from AMHS $(n=2)$.

\section{Transition Process}

Only three CAMHS teams and three AMHS teams had written transition guidelines. These teams were not from the same areas. The results reported in Table 1 regarding transition procedures suggest that the transition process itself is unstructured given that the majority of both CAMHS and AMHS teams reported a lack of agreed, accessible and known transition care arrangements. Furthermore, 56\% of CAMHS teams reported that a single clinician was always identified to co-ordinate the transition process but 54\% of AMHS teams reported that this was never done. Paper-based information exchange between services appears to be excellent as $97 \%$ of CAMHS teams and $86 \%$ of AMHS teams reported that a comprehensive summary of the CAMHS notes was always made available to the receiving service. However, joint working is limited. $60 \%$ of CAMHS teams reported only sometimes meeting with AMHS teams to discuss transition cases while $60 \%$ of AMHS teams reported this never happens. Furthermore, $57 \%$ of CAMHS teams and $80 \%$ of AMHS teams reported that they do not hold meetings involving 
staff from both services and the young person. Using chi-square analysis no differential patterns of response were observed across service type.

[Table 1 here]

Items relating to preparing young people for transition (see Table 2) suggested possible differences in services' perceptions of their role in transition. Chi square analysis revealed differential patterns of response across service type for two items. First, there was a significant association evident between service type and whether an AMHS clinician was identified to welcome the young person entering their care, $\chi^{2}(2, n=47)=12.75, p=.002$. Examination of the standardised residuals revealed that more AMHS clinicians than expected indicated that this always happens ( $\mathrm{SR}=2.0$ ) suggesting this cell is a major contributor to the overall chi square value. The opposite pattern was true for CAMHS clinicians. Only $4 \%$ of teams reported that this always happens.

Second, there was a significant association between service type and negotiation of the timing of transition with the young person, $\chi^{2}(2, n=46)=12.09, p=.002$. Examination of the standardised residuals revealed that more AMHS clinicians than expected indicated that this never happens $(\mathrm{SR}=2.2)$ suggesting this cell is a major contributor to the overall chi square value. The opposite pattern was true for CAMHS teams who were less likely to say it never happens.

Although these were not statistically significant, three other issues relating to differing views on the preparation of young people and carers are worth noting. First, 56\% of CAMHS teams reported that young people are always made familiar with the structure of the adult service, compared with $81 \%$ of AMHS teams, possibly indicating that this is seen as the role of AMHS rather than CAMHS. Second, $89 \%$ of CAMHS teams reported involving parents/carers in the care plan. The level of parental involvement in the young person's care plan varied from the perspective of AMHS teams with 52\% reporting this always happens and $44 \%$ reporting it 
as happening "sometimes". Finally, $65 \%$ of AMHS teams reported that they never teach the young person how to cope with transition. There was some variation in the extent to which this is done by CAMHS clinicians with only $39 \%$ reporting that this always happens. This could suggest that AMHS view this as falling under the remit of CAMHS.

On a more positive note, both services reported that they assist young people to take on a more adult role during transition with the majority of teams from both services reporting that they always discuss boundaries in confidentiality and always make a conscious effort to improve the factors supporting resilience in young people (such as discussing parental involvement).

[Table 2 here] 


\section{DISCUSSION}

Adolescent mental health must be prioritised to achieve the global agenda of improved mental health for all. ${ }^{21}$ This should include ensuring a seamless transition process between CAMHS and AMHS requiring individual countries to examine their health service structure . Our first aim in this study was to investigate the transition process in the Republic of Ireland. However, it is also worth commenting on transfers between CAMHS and AMHS as it has been suggested that both concepts should be investigated. ${ }^{3}$ Our transfer numbers give an indication of the number of young people who move between service annually rather than providing the number of successful transitions. The estimated annual numbers of young people who remained in CAMHS beyond the transitional boundary and those considered suitable for transfer were both greater than the estimated annual numbers transferred, a finding similar to that of Singh et al. ${ }^{9}$ We identified two potential barriers to transfer noted by Paul et al. ${ }^{3}$, namely, the refusal of a referral by AMHS and the absence of a referral from CAMHS based on the perceived lack of an appropriate adult service.

These findings suggest a potential gap in service provision for young people. This begs the question of what happens to young people who have a mental health service need but who are not referred to AMHS. ${ }^{3}$ Furthermore, the national variation in service cover for 16 and 17 year olds in our sample implies that while young people do receive a service, it is unlikely to be in an age-appropriate setting. Similar discrepancies have been linked in other countries to the traditional differences between CAMHS and AMHS such as differences in theoretical base, service organisation, and professional training. ${ }^{12}$

Our second aim was to compare best practice guidelines for transition with current process and clinical experience. The data suggest that optimal transitions are rare as found by Paul et al. ${ }^{3}$ While the data from the current study cannot inform us whether continuity of care was present, we found evidence of only one other element of successful transition, namely good 
information transfer. Our data indicated a relative absence of transition planning meetings and no period of parallel care.

There is a virtual absence of written protocols and policies to guide transition, a lack of formal planning, and limited face-to-face communication and collaboration between CAMHS and AMHS. The data suggest that transition in Ireland is not something that is seen as a process involving both CAMHS and AMHS, particularly in relation to preparing the young person for transition. These are similar findings to previous research which also noted a lack of fully developed, agreed transition policies ${ }^{13,14}$ and a need to develop dialogue between child and adult services. $^{7}$

This study is the first step in a comprehensive investigation of transition in Ireland and addresses the call to make youth mental health a global research priority. The findings provide the first clear indication of current operational practice with regards to transition, (given that over half of all CAMHS in the Republic of Ireland participated), and suggest areas for future service development. Our data indicate that similar issues exist in Ireland that have been identified elsewhere as potential barriers to successful transition, namely, variation in age-based service boundaries, ${ }^{7,8,13}$ differing service thresholds and eligibility requirements, ${ }^{9,10}$ and limited inter-agency communication and collaboration ${ }^{7}$

The current study does have some limitations. The first is the relatively low participation rate of AMHS teams despite repeated invitations to participate. Given that CAMHS and AMHS are not coterminous it was not possible to collect data from all AMHS that were in the same geographical areas as participating CAMHS. It is possible that transition is not a current AMHS service priority given that illnesses which would fit within this service are more likely to emerge during late adolescence and as noted earlier, first presentations at this age are more likely to go directly to AMHS rather than CAMHS, thereby not requiring transition. It is likely however that transition will increase in importance in the coming years as CAMHS takes on greater responsibility for the 16 to 18 year age group. ${ }^{22}$ 
A second limitation of this study was the reliance on self-report data around transitions and the lack of available essential clinical administrative data. It is noted that such problems have been reported in other published work and that these challenges to conducting research should be highlighted. ${ }^{3}$

Understanding the transition process is an initial step towards improving service delivery to young people. Our data reveal that Ireland shares the problems experienced worldwide when it comes to meeting the mental health needs of young people. However, it is hoped that the recent introduction of new national access protocols for 16 and 17 year olds requiring that all those under 18 are assessed and treated in CAMHS by January 2014 will improve service access for this age group and ensure all are treated in an age-appropriate setting. ${ }^{22}$

In addition to improving service access, continuity of care must be prioritised for those who may not meet AMHS criteria but who still have a mental health service need. Such cases should be documented clearly in order to make a case for future service development, particularly for young people for whom continuity via AMHS may not be the answer. ${ }^{3}$

Finally, transition should form part of professional training and inter-agency collaboration, a key element of successful transition, should be encouraged. Recommendations on best practice should be incorporated into formal healthcare policy. CAMHS and AMHS need to jointly develop shared policies for transition to provide a structure within which transition can take place and to provide clarity regarding the role of the professionals involved.

In conclusion, while this study provides an initial overview of the transition process in Ireland, additional qualitative data will be gathered in future ITRACK studies on transition experiences of young people themselves so that a comprehensive evidence base can be created to inform future service developments. Given that the issues and challenges identified thus far in Ireland are very similar to those reported in other countries, it is likely that these findings will assist in further developing services to meet the needs of young people worldwide. 


\section{ACKNOWLEDGEMENTS}

This research is funded by a Health Research Award from the Irish Health Research Board Grant No. HRA_HSR/2010/27. 


\section{REFERENCES}

1. McGorry PD. Transition to adulthood: The critical period for pre-emptive, diseasemodifying care for schizophrenia and related disorders. Schizophrenia Bull 2011; 37:524-530.

2. McGorry PD. The specialist youth mental health model: Strengthening the weakest link in the public mental health service. Med J Australia 2007; 187: S53-S56.

3. Paul M, Ford T, Kramer T, Islam Z, Harley K, Singh SP. Transfers and transitions between child and adult mental health services. Br J Psychiatry 2013; 202: s36-s40.

4. Blum RW, Garrell D, Hodgman CH, Jorissen TW, Okinow NA, Orr DP, et al. Transition from child-centred to adult health-care systems for adolescents with chronic conditions. A position paper of the Society for Adolescent Medicine. J Adolesc Health 1993; 14: 570-576.

5. Viner R. Transition from paediatric to adult care. Bridging the gap or passing the buck? Arch Dis Child 1999; 81: 271-273.

6. Singh SP. Transition of care from child to adult mental health services: The great divide. Curr Opin Psychiatry 2009; 22: 386-390.

7. Bruce H, Evans N. The transition into adult care. Psychiatr 2008; 7: 399-402.

8. While A, Forbes A, Ullman R, Lewis S, Mathes L, Griffiths P. Good practices that address continuity during transition from child to adult care: Synthesis of the evidence. Child Care Health Dev 2004; 30: 439-452.

9. Singh SP, Paul M, Ford T, Kramer T, Weaver T, McLaren S, et al. Process, outcome and experience of transition from child to adult mental healthcare: Multiperspective study. Br J Psychiatry 2010; 197: 305-312.

10. Tantam D. Pathways into adult care. Psychiatry 2005; 4: 141-144. 
11. Muñoz-Solomando A, Townley A, Williams R. Improving transitions for young people who move from child and adolescent mental health services to mental health services for adults: Lessons from research and young people's and practitioners' experiences. Curr Opin Psychiatry 2010; 23: 311-317.

12. Singh SP, Evans N, Sireling L, Stuart H. Mind the gap: The interface between child and adult mental health services. The Psychiatrist 2005; 29: 292-294.

13. Singh SP, Paul M, Ford T, Kramer T, Weaver T. Transitions of care from child and adolescent mental health services to adult mental health services (TRACK study): A study of protocols in Greater London. BMC Health Serv Res 2008; 8: 135-141.

14. Williams J, Hewson L. Improving the transition from CAMHS to adult services. YoungMinds Magazine 2010; 103: 13.

15. Pottick KJ, Bilder S, Van der Stoep A, Warner LA, Alvarez MF. US patterns of mental health service utilisation for transition-age youth and young adults. J Behav Health Ser $R$ 2008; 35: 373-389.

16. Hovish K, Weaver T, Islam Z, Paul M, Singh SP. Transition experiences of mental health service users, parents and professionals in the United Kingdom: A qualitative study. Psychiatr Rehabil J 2012; 35: 251-257.

17. Davidson, S., Cappelli, M., \& Vloet, M.A. (2011). We've got growing up to do: Transitions policy paper. [monograph on the Internet]. Ontario Centre of Excellence for Child and Youth Mental Health Ontario Canada: Pub; 2011 [cited 2012 Dec 6]. Available from: www.excellenceforchildandyouth.ca/sites/default/files/policy_growing_up_to_do.pdf

18. Department of Health and Children. A Vision for Change: Report of the Expert Group on Mental Health Policy [monograph on the Internet]. Dublin, Ireland: Government Publications Office; 2006 [cited 2012 Dec 6]. Available from: www.dohc.ie/publications/vision_for_change.html 
19. Department of Health and Children. Second Report of the Working Group on Child \& Adolescent Psychiatric Services. [monograph on the Internet]. Dublin, Ireland: Department of Health \& Children; 2003 [cited 2012 Dec 6]. Available from: www.dohc.ie/publications/second_report_of_the_working_group_on_child_and_adoles cent_psychiatric_services.html

20. Royal College of Nursing. Lost in transition: Moving young people between child and adult health services [monograph on the Internet]. London: Royal College of Nursing; 2007 [cited 2012 Oct 15]. Available from: www.rcn.org.uk/_data/assets/pdf_file/0010/157879/003227.pdf

21. Sawyer SM, Afifi RA, Bearinger LH, et al. Adolescence: A foundation for future health. Lancet 2012; 379: 1630-1640.

22. Specialist CAMHS Advisory Group. Fourth annual child and adolescent mental health services report 2011-2012 [monograph on the Internet]. Dublin, Ireland: Health Service Executive; 2012 [cited 2012 Dec 6]. Available from:

www.hse.ie/eng/services/Publications/services/Mentalhealth/camhs20112012annualrep ort.pdf 